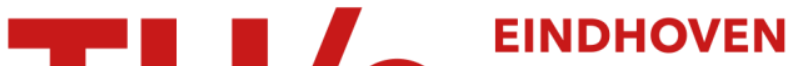 \\ UNIVERSITY OF \\ TECHNOLOGY
}

\section{The electrostatic field of a point charge inside a cylinder, in connection with wave guide theory}

\section{Citation for published version (APA):}

Bouwkamp, C. J., \& Bruijn, de, N. G. (1947). The electrostatic field of a point charge inside a cylinder, in connection with wave guide theory. Journal of Applied Physics, 18(6), 562-577.

\section{Document status and date:}

Published: 01/01/1947

\section{Document Version:}

Publisher's PDF, also known as Version of Record (includes final page, issue and volume numbers)

\section{Please check the document version of this publication:}

- A submitted manuscript is the version of the article upon submission and before peer-review. There can be important differences between the submitted version and the official published version of record. People interested in the research are advised to contact the author for the final version of the publication, or visit the $\mathrm{DOI}$ to the publisher's website.

- The final author version and the galley proof are versions of the publication after peer review.

- The final published version features the final layout of the paper including the volume, issue and page numbers.

Link to publication

\section{General rights}

Copyright and moral rights for the publications made accessible in the public portal are retained by the authors and/or other copyright owners and it is a condition of accessing publications that users recognise and abide by the legal requirements associated with these rights.

- Users may download and print one copy of any publication from the public portal for the purpose of private study or research.

- You may not further distribute the material or use it for any profit-making activity or commercial gain

- You may freely distribute the URL identifying the publication in the public portal.

If the publication is distributed under the terms of Article 25fa of the Dutch Copyright Act, indicated by the "Taverne" license above, please follow below link for the End User Agreement:

www.tue.nl/taverne

Take down policy

If you believe that this document breaches copyright please contact us at:

openaccess@tue.nl

providing details and we will investigate your claim. 
assumption mentioned above, occurs, is consequently not negligible compared with the total amount of heat accumulated in the conductor. The preparation of a more exact theory, as mentioned above, seems to be justified.
The work here presented has been carried out at Sieverts Kabelverk, Sundbyberg, Sweden. I thank Sieverts Kabelverk and especially $\mathrm{Mr}$. H. Spanne, chief engineer at Sieverts Kabelverk, for permission to publish this paper.

\title{
The Electrostatic Field of a Point Charge Inside a Cylinder, in Connection with Wave Guide Theory
}

\author{
C. J. Bouwkamp and N. G. DE Bruijn \\ Natuurkundig Laboratorium der N. V. Philips' Gloeilampenfabrieken, Eindhoven, Netherlands
}

(Received February 21, 1947)

In this paper the field of a point charge inside a hollow, infinitely long circular cylinder is studied. The case of an axial point charge is treated in detail. Three different methods are developed. The first method shows how to calculate the induced charges at the surface of the cylinder without explicit knowledge of the potential itself. The surface charge-density function is obtained as the solution of a Fourier-type integral equation. Then the potential caused by these charges is calculated. The second method works in the opposite direction. Here the potential is obtained as solution of a boundary value problem, followed by the calculation of the corresponding charges at the surface of the cylinder.

The integral, obtained for the surface charge density, is transformed by contour integration. Although the resulting series is very useful for numerical purposes, a stronger method is necessary, in order to calculate the charge density just opposite the point source. Figure 1 shows the calculated values of the induced surface charge density. Several approximations for the charge-density function are

\section{INTRODUCTION}

$\mathrm{P}^{\mathrm{r}}$ REVIOUS works of Schelkunoff, ${ }^{1}$ Weyrich, ${ }^{2}$ Buchholz, ${ }^{3}$ and Oberhettinger, ${ }^{4}$ among others, already contain complete information with regard to fields in circular pipes, as produced by Hertzian dipoles. Therefore, the generation.

1S. A. Schelkunoff, "Modified Sommerfeld's integral and its applications," Proc. I.R.E. 24, 1388 (1936); and "Transmission theory of plane electromagnetic waves," ibid. 25, 1457 (1937); with bibliography.

${ }^{2} \mathrm{R}$. Weyrich, "Ueber einige Randwertprobleme, insbesondere der Elektrodynamik," J. reine angew. Math. 172, 133 (1934).

${ }^{3} \mathrm{H}$. Buchholz, "Gekoppelte Strahlungsfelder im kreiszylindrischen Hohlleiter," Ann. d. Physik 39, 81 (1941).

${ }^{4} \mathrm{~F}$. Oberhettinger, "Ueber ein Randwertproblem der Wellengleichung in Zylinderkoordinaten," Ann. d. Physik 43, 136 (1943). considered, in connection with recent work of Weber (cf. Table I).

The same is done for the field inside the cylinder. Various formulas are given which allow of numerical calculations. In Table II some calculated values of the potential are shown.

The third method is based on the theory of FourierBessel-Dini series. The potential is developed in terms of discrete normal solutions of the potential equation in cylindrical coordinates. The coefficients in this development can be derived from the behavior of the potential in the immediate neighborhood of the primary source.

Furthermore, it is emphasized that the study of the above potential problem can serve as a guide in questions of wave propagation in hollow circular cylinders. In this connection the third method is shown to be extremely useful, as it enables us to calculate directly the fields in the far zone from that in the immediate neighborhood of the exciting source. This new method is demonstrated in case of acoustic waves inside a cylinder, caused by a harmonically vibrating point source.

of special types of wave patterns, caused by given current and charge distributions on the exiting antennas, can be dealt with quantitatively. The calculations in this domain of electromagnetics, however, are far from elementary, such that a non-mathematically minded engineer might hardly recognize the usefulness of those theoretical treatments. Hence, every attempt to diminish the necessary amount of mathematics, may be worth while to consider. Until now a considerable amount of complex function theory is required. We believe, however, that this can be greatly avoided if one bases oneself on the theory of Fourier-Bessel-Dini series. This method is undoubtedly within reach of every engineer, 
because the analogous conventional theory of the ordinary Fourier series is common knowledge nowadays.

In this paper we will not consider the vector wave propagation as occurring in electromagnetic theory. Those topics will be treated elsewhere. The new method will be demonstrated in the case of scalar wave propagation only.

The major part of the present article, however, will be devoted to the study of the electrostatic field of a point charge inside the cylinder. Consequently, only a minor part of the mathematics developed here is claimed to be useful in wave guide theory.

Beginning with the simplest problem, we confine ourselves to the case of a point charge situated on the axis of the cylinder. Later on we will briefly indicate how to extend the theory to excentric point charges.

\section{STATEMENT OF THE SIMPLEST PROBLEM. CALCULATION OF THE CHARGES INDUCED ON THE SURFACE OF THE CYLINDER}

Let $a$ be the radius of the cylinder, and let the point charge have a value +1 . Furthermore, let $z, \rho, \varphi$ denote the right-handed cylindrical coordinates of a field point $P$. Henceforth the origin may coincide with the given point charge, and the $z$-axis be taken along the axis of the cylinder.

The electric force $\mathbf{E}$ can be derived from a potential $V$ according to $\mathbf{E}=-\operatorname{grad} V$. It is evident that the $z$-axis is an axis of symmetry, so that $V$ depends only on $z$ and $\rho$. In addition, $V$ is even in $z$.

To calculate $\mathbf{E}$ and $V$, we first follow a rather unconventional method, by calculation of the charges, induced on the wall of the cylinder, without explicit knowledge of the potential itself. Afterwards the field is readily found from the original point charge and these induced charges. The surface density of the induced charges will be denoted by $\eta(z)$.

We may remark that $V$ is the so-called Green function of the first kind. It is a regular solution of the potential equation, except for a singularity in the origin, so that the function $V(P)-1 / r$ remains finite for $r \rightarrow 0$; furthermore, $V=0$ on the boundary, and both $V$ and $\operatorname{grad} V$ tend to zero, if $P$ tends to infinity inside the cylinder.
As is well known, there is one, and only one, function $V(z, \rho)$ fulfilling all these conditions simultaneously. Once this function is obtained, the charges on the boundary of the cylinder can be computed from

$$
\eta(z)=\frac{1}{4 \pi} \frac{\partial V}{\partial \rho}, \quad(\rho=a) .
$$

Let us now show how $\eta(z)$ can be found without explicit knowledge of the potential $V$. Be $g(z, \rho)$ a given regular solution of the potential equation inside the cylinder, and suppose $g$ and $\partial g / \partial z$ to be bounded in that domain. By Green's theorem, we at once express the value of $g$ in the origin as a surface integral over its boundary values, namely

$g(0,0)=-\frac{1}{4 \pi} \int g \frac{\partial V}{\partial \rho} d \sigma=-\int g(z, a) \eta(z) d \sigma$.

In this $d \sigma$ means the surface element of the boundary of the cylinder; the integration in (2) extends over this boundary.

Omitting the left-hand integral in (2), the remaining equality can be interpreted as an integral equation for $\eta(z)$, which may be readily solved, if a convenient choice is made for the rather arbitrary function $g$. We take

$$
g(z, \rho)=e^{-i \lambda z} I_{0}(\lambda \rho)
$$

where $I_{0}$ is the Bessel function of purely imaginary argument and zeroth order. The function (3) is a regular potential, with $g$ and $\partial g / \partial z$ bounded inside the cylinder, if the constant $\lambda$ is restricted to real values.

Substitution of (3) in (2) leads to

$$
1=-\int_{0}^{2 \pi} a d \varphi \int_{-\infty}^{\infty} e^{-i \lambda z} I_{0}(\lambda a) \eta(z) d z
$$

whence it follows

$$
\int_{-\infty}^{\infty} z^{-i \lambda z} \eta(z) d z=-\frac{1}{2 \pi a I_{0}(\lambda a)},(-\infty<\lambda<\infty) .
$$

Thus the integral equation (2) is reduced to one of the common Fourier type (4). Its solution can be written down immediately. By inversion of 
(4), one obtains for the required charge density

$$
\begin{array}{r}
\eta(z)=-\frac{1}{4 \pi^{2} a} \int_{-\infty}^{\infty} e^{i \lambda z} \frac{d \lambda}{I_{0}(\lambda a)} \\
=-\frac{1}{2 \pi^{2} a^{2}} \int_{0}^{\infty} \frac{\cos (z / a) t}{I_{0}(t)} d t .
\end{array}
$$

Starting from this integral expression, we can develop solutions more useful for numerical purposes. For non-vanishing $z$ we may transform the above integral into a series by means of the conventional contour integration. In the cut plane $z=0$, however, stronger methods must be used, as will be seen below.

Consider the following integral in the complex $t$-plane:

$$
\frac{1}{2 \pi i} \int_{W} \frac{e^{i z t / a}}{I_{0}(t)} d t
$$

the path of integration $W$ leading from $-R$ to $R$ along the real axis, and back from $R$ to $-R$ along a semi-circle of radius $R$ and center 0 . Avoiding values of $R$ for which the semi-circle passes through a zero of $I_{0}(t)$, we can prove that for positive values of $z$, the contribution of the semi-circle tends to zero, when $R$ tends to infinity. Let $k_{1}, k_{2}, \cdots$ denote, in ascending order, the positive zeros of the Bessel function $J_{0}(k)$. Then $i k_{n}$ are the simple poles of the integrand in the upper half of the $t$-plane. The residue theorem now gives

$$
\frac{1}{2 \pi i} \int_{-\infty}^{\infty} \frac{e^{i z t / a}}{I_{0}(t)} d t=\sum_{n=1}^{\infty} \frac{e^{-k_{n} z / a}}{I_{0}^{\prime}\left(i k_{n}\right)} .
$$

We have $I_{0}^{\prime}\left(i k_{n}\right)=i J_{1}\left(k_{n}\right)$, and so for the charge density

$$
\eta(z)=-\frac{1}{2 \pi a^{2}} \sum_{n=1}^{\infty} \frac{e^{-k_{n} z / a}}{J_{1}\left(k_{n}\right)}, \quad(z \geqslant 0) .
$$

Only for $z=0$ does this series not converge in the ordinary sense. Unless $z$ is very small, it is very useful for numerical computation. A small number of terms are already sufficient in the range $z \geqslant a$.

The total charge, induced on the cylinder between $z \geqslant 0$ and $\infty$, can be found by integra- tion :

$$
2 \pi a \int_{z}^{\infty} \eta(\zeta) d \zeta=-\sum_{n=1}^{\infty} \frac{e^{-k_{n} z / a}}{k_{n} J_{1}\left(k_{n}\right)}, \quad(z \geqslant 0) .
$$

The latter series does converge at $z=0$. The total amount of induced charge on the upper half of the cylinder should be $-\frac{1}{2}$; we have thus an opportunity to verify a well-known formula in Bessel-function theory, originally credited to Nielsen :

$$
\sum_{n=1}^{\infty} \frac{1}{k_{n} J_{1}\left(k_{n}\right)}=\frac{1}{2}
$$

For small values of $z$ the convergence of the series (6) is too slow for practical evaluation. Hence we have to develop other expressions for $\eta(z)$, which do allow of numerical computation near the "cut plane" $z=0$. This can be accomplished by Watson's method for an analogous problem. ${ }^{5}$

Therefore we consider the following integral

$$
\frac{1}{2 \pi i} \int \frac{\cos \lambda w}{(w-t) I_{0}(w) \cos (\pi w / \beta)} d w
$$

wherein $\lambda, \beta, t$ are real parameters; $\beta>0$, $\cos (\pi t / \beta) \neq 0$. The path of integration is a circle of radius $R>t$, and center 0 , not passing through any zero of the denominator of the integrand. When one adds the condition $\beta|\lambda|<\pi$, the integral tends to zero, if $R$ increases to infinite values. Thus the sum of the residues of the integrand is zero also. The poles of the integrand are all simple; they lie at

$$
\begin{aligned}
& w=t, \\
& w= \pm i k_{n}, \quad(n=1,2, \cdots), \\
& w= \pm\left(\nu+\frac{1}{2}\right) \beta, \quad(\nu=0,1,2, \cdots) .
\end{aligned}
$$

The corresponding residues are easily found to be

$$
\begin{gathered}
\frac{\cos \lambda t}{I_{0}(t) \cos (\pi t / \beta)}, \\
-\frac{\cosh \lambda k_{n}}{J_{1}\left(k_{n}\right) \cosh \left(\pi k_{n} / \beta\right)\left[k_{n} \pm i t\right]}, \\
-\frac{(-1) \nu \cos (\lambda \beta \nu+\lambda \beta / 2)}{\pi} .
\end{gathered}
$$

"G. N. Watson, "The use of series of Bessel functions in problems connected with cylindrical wind-tunnels," Proc. Roy. Soc. 130, 29 (1930/31). 


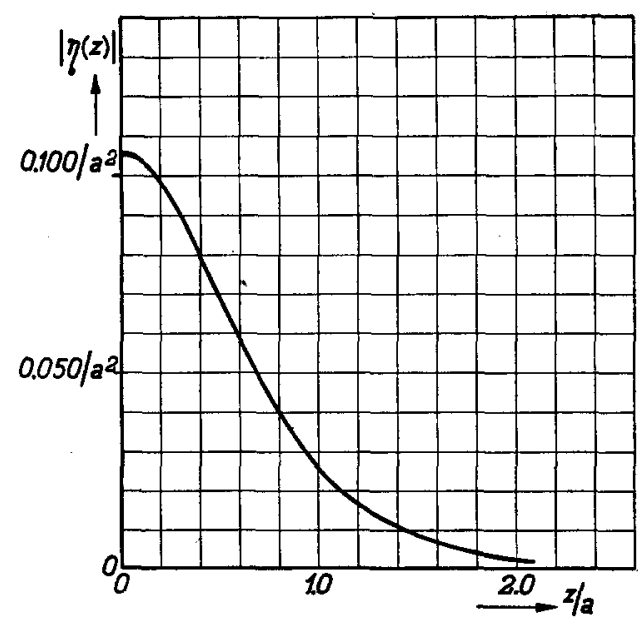

FIG. 1. Curve showing the surface charge density $|\eta|$ on the wall of the grounded cylinder, induced by a unit-point charge situated in the axis at $z=0 ; z$ denotes the distance along the cylinder, measured from the source containing plane; $a$ is the radius of the cylinder.

The sum of all residues together being zero, one has

$$
\begin{gathered}
\frac{\cos \lambda t}{I_{0}(t) \cos (\pi t / \beta)}=\sum_{n=1}^{\infty} \frac{2 k_{n} \cosh \lambda k_{n}}{J_{1}\left(k_{n}\right) \cosh \left(\pi k_{n} / \beta\right)} \frac{1}{k_{n}^{2}+t^{2}} \\
+\frac{\beta}{\pi} \sum_{\nu=0}^{\infty} \frac{(-1)^{\nu} \cos (\lambda \beta \nu+\lambda \beta / 2)}{I_{0}(\beta \nu+\beta / 2)} \frac{(2 \nu+1) \beta}{(\beta \nu+\beta / 2)^{2}-t^{2}}
\end{gathered}
$$

Evidently, the right-hand side of $(7)$ is nothing else but the expansion in rational fractions of the left-hand side.

We now multiply both sides of $(7)$ by $\cos (\pi t / \beta)$, thus neutralizing the poles in (c), and integrating from $t=-\infty$ to $t=\infty$. Using the following formulas ${ }^{5}$

$$
\begin{array}{r}
\int_{-\infty}^{\infty} \frac{\cos (\pi t / \beta)}{k_{n}^{2}+t^{2}} d t=\frac{\pi}{k_{n}} e^{-\pi k_{n} / \beta} \\
\int_{-\infty}^{\infty} \frac{\cos (\pi t / \beta)}{(\beta \nu+\beta / 2)^{2}-t^{2}} d t=\frac{(-1)^{\nu} 2 \pi}{(2 \nu+1) \beta}
\end{array}
$$

we easily obtain

$$
\begin{aligned}
\int_{0}^{\infty} \frac{\cos \lambda t}{I_{0}(t)} d t=\beta \sum_{\nu=0}^{\infty} & \frac{\cos \lambda \beta\left(\nu+\frac{1}{2}\right)}{I_{0}(\beta \nu+\beta / 2)} \\
& +2 \pi \sum_{n=1}^{\infty} \frac{\cosh \lambda k_{n}}{J_{1}\left(k_{n}\right)\left[1+e^{2 \pi k_{n} / \beta}\right]}
\end{aligned}
$$

Hence, for the induced charge density

$$
\begin{aligned}
-a^{2} \eta(z)=\frac{\beta}{2 \pi^{2}} \sum_{\nu=0}^{\infty} & \frac{\cos (\beta \nu+\beta / 2) z / a}{I_{0}(\beta \nu+\beta / 2)} \\
& +\frac{1}{\pi} \sum_{n=1}^{\infty} \frac{\cosh k_{n} z / a}{J_{1}\left(k_{n}\right)\left[1+e^{2 \pi k_{n} / \beta}\right]} .
\end{aligned}
$$

It may be emphasized that our proof of (10) holds for $\beta>0$ and $-\pi a / \beta<z<\pi a / \beta$. The first series on the right of $(10)$ is convergent for all real values of $z$. It may be considered as an approximation of the integral in (5), obtained when the integration interval is divided into equal parts of length $\beta$, and the integrand in each separate interval is replaced by its central value. The second series should then be interpreted as a correction term. The latter, however, only converges for $-2 \pi a / \beta<z<2 \pi a / \beta$. By analytic continuation, it can be shown that (10) holds for this extended range, twice as large as might be expected from the proof of (10), as given above. Moreover, for the special value $\beta=a \pi /|z|$, the first series vanishes identically, whilst the "correction term" reduces to the series (6). Consequently, Eq. (10) is more basic than (6).

Equation (10) is very suitable for numerical calculations. The constant $\beta$ is still arbitrary, except for $-2 \pi a / \beta<z<2 \pi a / \beta$. Let us consider the extreme case $z=0$ in detail. We already saw that in this case (6) cannot be used on behalf of its divergence. Equation (10) can be used with any positive $\beta$. We calculated $-2 \pi^{2} a^{2} \eta(0)$ in seven decimals with $\beta=1, \beta=3$, respectively. In the first case we had to take eighteen terms of the first series and only one of the second. The result was found to be

$$
2.0832296+0.0000033=2.0832329 .
$$

With $\beta=3$ we had to consider six terms of the first, and three terms of the second series, obtaining in this case

$$
2.0053020+0.0779307=2.0832327,
$$

in close agreement with the earlier value. Thus we can state

$\int_{0}^{\infty} \frac{d t}{I_{0}(t)}=2.083232 \cdots, \quad \eta(0)=-\frac{0.10554}{a^{2}}$ 
Numerical computation of the surface charge density is now easily established. When $z \geqslant a$, already the first four terms of (6) are sufficient to guarantee the correctness of the fourth decimal. Inserting known data from existing Bessel-function tables, (6) becomes

$$
\begin{aligned}
-a^{2} \eta(z)= & 0.30657 \exp (-2.40 z / a) \\
& -0.46774 \exp (-5.52 z / a) \\
& +0.58636 \exp (-8.65 z / a) \\
& -0.68466 \exp (-11.79 z / a) .
\end{aligned}
$$

In the remaining range $0 \leqslant z \leqslant a$, Eq. (10) may be used with $\beta=2$. Then one obtains

$$
\begin{gathered}
-a^{2} \eta(z)=0.08003 \cos (z / a)+0.02076 \cos (3 z / a) \\
+0.00372 \cos (5 z / a)+0.00060 \cos (7 z / a) \\
+0.00009 \cos (9 z / a)+0.00001_{4} \cos (11 z / a) \\
+0.00032 \cosh (2.4 z / a)
\end{gathered}
$$

The data of Fig. 1 were computed by means of Eqs. (12) and (13). The curve shows a rapid decrement of the surface charges with increasing distance from the cut plane.

By expanding both sides of (9) into a power series of $\lambda$, and identifying the corresponding coefficients, we obviously obtain

$$
\begin{aligned}
\int_{0}^{\infty} \frac{t^{2 r}}{I_{0}(t)} d t=\beta \sum_{\nu=0}^{\infty} & \frac{(\beta \nu+\beta / 2)^{2 r}}{I_{0}(\beta \nu+\beta / 2)} \\
& +(-1)^{r} 2 \pi \sum_{n=1}^{\infty} \frac{k_{n}{ }^{2 r}}{J_{1}\left(k_{n}\right)\left[1+e^{2 \pi k_{n} / \beta}\right]} .
\end{aligned}
$$

The numerical values of these integrals can be found in the same way as shown above for $r=0$. Then for $\eta(z)$ a power series in $z^{2} / a^{2}$ can be derived, convergent for $|z|<a$. The expression (13), however, is much more useful for numerical purposes in the corresponding range of $z$-values.

\section{FORMULAS FOR THE POTENTIAL AND THE ELECTRIC FIELD}

The potential $V(P)$ can be considered as the sum of the potential $1 / r$, due to the unit charge in the origin, and the potential $V_{0}(P)$, due to the induced charges on the surface of the cylinder:

$$
V(P)=\frac{1}{r}+V_{0}(P)=\frac{1}{r}+\int \frac{\eta(\zeta)}{r_{1}} d \sigma .
$$

In this $r, r_{1}$, denote the distances from $P$ to the origin, and the surface element, respectively. Again, $V_{0}(P)$ depends only upon $z$ and $\rho$.

At first we restrict ourselves to points on the axis of the cylinder. Then we have

$$
V_{0}(z, 0)=2 \pi a \int_{-\infty}^{\infty} \frac{\eta(x+u)}{\left(u^{2}+a^{2}\right)^{\frac{1}{2}}} d u .
$$

Using (5), we obtain

$$
V_{0}(z, 0)=-\frac{2}{\pi} \int_{0}^{\infty} \frac{\cos \lambda z}{I_{0}(\lambda a)} d \lambda \int_{0}^{\infty} \frac{\cos \lambda u}{\left(u^{2}+a^{2}\right)^{\frac{1}{2}}} d u .
$$

On account of a well-known integral for the $K$-function, namely

$$
K_{0}(\lambda a)=\int_{0}^{\infty} \frac{\cos \lambda u}{\left(u^{2}+a^{2}\right)^{\frac{1}{2}}} d u,
$$

we obtain for the potential of the induced charges, at points on the axis of the cylinder

$$
V_{0}(z, 0)=-\frac{2}{\pi} \int_{0}^{\infty} \frac{K_{0}(\lambda a)}{I_{0}(\lambda a)} \cos \lambda z d \lambda .
$$

In the rotationally symmetrical problem under consideration, the potential outside the axis is easily obtained, once the potential on the axis of symmetry has been found. We simply have to take the mean value of $V_{0}(z+i \rho \cos \varphi, 0)$ over a full period of the angle $\varphi$. Thus

$$
V_{0}(z, \rho)=\frac{1}{2 \pi} \int_{0}^{2 \pi} V_{0}(z+i \rho \cos \varphi, 0) d \varphi .
$$

The integration over $\varphi$ can be performed, leading to

$$
V_{0}(z, \rho)=-\frac{2}{\pi} \int_{0}^{\infty} \frac{K_{0}(\lambda a)}{I_{0}(\dot{\lambda} a)} I_{0}(\lambda \rho) \cos \lambda z d \lambda,
$$

and for the total potential we get

$$
\begin{aligned}
V(z, \rho)=\frac{1}{\left(z^{2}+\rho^{2}\right)^{\frac{3}{2}}}- & \frac{2}{\pi a} \int_{0}^{\infty} \frac{K_{0}(t)}{I_{0}(t)} \\
& \times I_{0}(t \rho / a) \cos (1 z / a) d t .
\end{aligned}
$$

The corresponding axial and radial components of the electric force are readily derived from 
(18) by differentiation

$$
\begin{aligned}
E_{z}= & \frac{z}{\left(z^{2}+\rho^{2}\right)^{\frac{1}{2}}}-\frac{2}{\pi a^{2}} \int_{0}^{\infty} \frac{K_{0}(t)}{I_{0}(t)} \\
& \times t I_{0}(t \rho / a) \sin (t z / a) d t \\
E_{\rho}= & \frac{\rho}{\left(z^{2}+\rho^{2}\right)^{\frac{3}{2}}}+\frac{2}{\pi a^{2}} \int_{0}^{\infty} \frac{K_{0}(t)}{I_{0}(t)} t I_{1}(t \rho / a) \cos (t z / a) d t
\end{aligned}
$$

It does not seem possible to transform these integrals exṕlicitly into known functions.

Having thus found expressions for the electric field inside the cylinder, we can easily verify them afterwards. For any real value of $\lambda$, the function

$$
\cos (\lambda z) \cdot I_{0}(\lambda \rho)
$$

is a regular solution of the potential equation inside the cylinder. Furthermore, the right-hand side of (17), being an absolutely convergent linear combination of those functions, is here a regular potential as well. Therefore, the function $V$ of (18) is also a regular potential, with exception of the origin, where it has just the required singularity. It only remains to be checked that $V$ vanishes on the boundary at $\rho=a$. This, however, is evident from the Fourier inversion of (14):

$$
\frac{2}{\pi} \int_{0}^{\infty} K_{0}(\lambda a) \cos \lambda z d \lambda=\frac{1}{\left(z^{2}+a^{2}\right)^{\frac{1}{2}}} .
$$

\section{A SECOND METHOD TO SOLVE THE POTENTIAL PROBLEM'}

The first method to obtain expressions for the field inside the cylinder, as given above, started from a certain pre-calculated surface charge distribution on the boundary. This method is a little unconventional. Usually one works in the opposite direction : first the potential is obtained as the solution of a boundary value problem, and then the surface charges are calculated. The results above immediately indicate how this second method runs. One should try to find a function $f(\lambda)$ such that the potential of the yet

6E. Weber, "The electrostatic field produced by a point charge in the axis of a cylinder," J. App. Phys. 10, $663(1939)$. unknown charges be expressible as follows

$$
V_{0}(z, \rho)=\int_{0}^{\infty} f(\lambda) I_{0}(\lambda \rho) \cos \lambda z d \lambda,
$$

this being a continuous sum of elementary potential functions. Fortunately, the primary potential of the unit point charge can already be expressed in that way:

$$
\frac{1}{r}=\frac{2}{\pi} \int_{0}^{\infty} K_{0}(\lambda \rho) \cos \lambda z d \lambda
$$

and, hence, for the total potential

$$
V(z, \rho)=\int_{0}^{\infty}\left\{f(\lambda) I_{0}(\lambda \rho)+\frac{2}{\pi}-K_{0}(\lambda \rho)\right\} \cos \lambda z d \lambda .
$$

A sufficient condition that $V(z, \rho)$ be zero on the boundary of the cylinder is that the integrand vanish there; consequently the function $f(\lambda)$ can be determined from

$$
f(\lambda) I_{0}(\lambda a)+\frac{2}{\pi} K_{0}(\lambda a)=0,
$$

and then one obtains (17).

Moreover, in combining Eqs. (17) and (20), we can derive the following expression

$$
\begin{array}{r}
V(z, \rho)=\frac{2}{\pi} \int_{0}^{\infty} \frac{I_{0}(\lambda a) K_{0}(\lambda \rho)-K_{0}(\lambda a) I_{0}(\lambda \rho)}{I_{0}(\lambda a)} \\
\times \cos \lambda z d \lambda,
\end{array}
$$

which more directly shows the vanishing at $\rho=a$. Equation (21) enables us to come to the integral expression (5) for the charge density. Theretofore, we use (1) and the Wronskian of $I_{0}, K_{0}$ :

$$
I_{0}(t) K_{0}{ }^{\prime}(t)-I_{0}^{\prime}(t) K_{0}(t)=-\frac{1}{t}
$$

and so we are back at our former starting point.

\section{APPROXIMATE EXPRESSIONS FOR THE SURFACE CHARGES}

Weber, ${ }^{6}$ in his theory of a point charge inside a cylinder, follows the second method apart from slight modifications of minor importance. His Eqs. (13), (17), and (18) are equivalent to ours as shown in Eqs. (18) and (19). Equation (5) for 
the surface charge density, however, was not given by Weber.

As for numerical calculations, Weber writes "the actual evaluation of the field distribution is rather difficult on account of the Fourier integrals which necessitate a point for point numerical integration in infinite limits," and further "the numerical evaluation of the integrals is very laborious." Weber, therefore, does not integrate point for point, but approximates the integrands by more tractable elementary functions, such that the remaining integrals can be computed explicitly.

As a fair approximation, suggested by existing tables of Bessel functions, Weber uses, for instance

$$
\frac{K_{0}(t)}{I_{0}(t)} t I_{1}(t) \approx \frac{t}{2} e^{-t}, \quad(t>0) .
$$

From the lower integral in (19), $E_{\rho}$ can then be evaluated at the surface of the cylinder. Weber's result for the corresponding density of charge, according to $\eta(z)=-E_{\rho} / 4 \pi$, may be written

$$
\begin{array}{r}
-a^{2} \eta(z) \approx \frac{1}{4 \pi}\left\{\frac{1}{\left(1+\zeta^{2}\right)^{\frac{3}{2}}}+\frac{1}{\pi} \frac{1-\zeta^{2}}{\left(1+\zeta^{2}\right)^{2}}\right\}, \\
(\zeta=z / a) .
\end{array}
$$

Furthermore, Weber derives expressions for the field in the cut plane as well for points at the surface of the cylinder, by another approximation, different from (23). His approximation (21) reads

$$
\frac{K_{0}(t)}{I_{0}(t)} \approx(2 \pi)^{t} t e^{-2 t}, \quad(t>0) .
$$

We need not, however, confine ourselves to the special values $z=0$ or $\rho=a$, as did Weber. When (24) is applied, we obtain

$$
\begin{aligned}
& \frac{1}{(2 \pi)^{1}} \int_{0}^{\infty} \frac{K_{0}(t)}{I_{0}(t)} I_{0}(t \rho / a) \cos (t z / a) d t \\
& \quad \approx \operatorname{Re} \int_{0}^{\infty} e^{-t(2+i \zeta)} J_{0}(i t \rho / a) t d t \\
& \\
& \approx \operatorname{Re} \frac{2+i \zeta}{\left[(2+i \zeta)^{2}-\rho^{2} / a^{2}\right]^{\frac{3}{3}}}
\end{aligned}
$$

TABLE I. Values of surface charge density $|\eta|$ in units $1 / a^{2}$. First column: rigorous values; second: Weber's approximation $\left(23^{\prime}\right)$. Third and fourth columns: approximations $\left(25^{\prime}\right),\left(24^{\prime}\right)$, respectively.

\begin{tabular}{lllll}
\hline$z / a$ & \multicolumn{4}{c}{$-a^{2} \eta(z)$} \\
\hline 0 & 0.1055 & 0.1049 & 0.1040 & 0.1285 \\
0.2 & 0.0980 & 0.0975 & 0.0969 & 0.1144 \\
0.4 & 0.0795 & 0.0795 & 0.0792 & 0.0827 \\
0.6 & 0.0581 & 0.0589 & 0.0586 & 0.0510 \\
0.8 & 0.0396 & 0.0413 & 0.0406 & 0.0280 \\
1.0 & 0.0259 & 0.0281 & 0.0269 & 0.0147 \\
1.2 & 0.0165 & 0.0190 & 0.0174 & 0.0076 \\
1.4 & 0.0104 & 0.0129 & 0.0111 & 0.0041 \\
1.6 & 0.0065 & 0.0087 & 0.0069 & 0.0025 \\
1.8 & 0.0040 & 0.0060 & 0.0042 & 0.0018 \\
2.0 & 0.0025 & 0.0041 & 0.0025 & 0.0016 \\
\hline \hline
\end{tabular}

and then, from (18)

$V(z, \rho) \approx \frac{1}{\left(z^{2}+\rho^{2}\right)^{\frac{1}{3}}}-\frac{1}{a}\left(\frac{8}{\pi}\right) \operatorname{Re} \frac{2+i \zeta}{\left[(2+i \zeta)^{2}-\rho^{2} / a^{2}\right]^{\frac{3}{3}}}$.

Especially on the cut plane, when differentiated to $(-\rho)$, this formula yields

$$
E_{\rho} \approx \frac{1}{\rho^{2}}\left\{1+\left(\frac{8}{\pi}\right)^{\frac{1}{2}} \frac{6 \rho^{3} / a^{3}}{\left[4-\rho^{2} / a^{2}\right]^{5 / 2}}\right\}, \quad(z=0) .
$$

This result is apparently in disagreement with that of Weber. His relation reads

$$
E_{\rho, \text { Weber }} \approx \frac{1}{\rho^{2}}\left\{1+\left(\frac{8}{\pi}\right)^{\frac{1}{2}} \frac{\rho^{3} / a^{3}}{\left[4-\rho^{2} / a^{2}\right]^{\frac{3}{3}}}\right\}, \quad(z=0) .
$$

The reason for this discrepancy is that Weber dropped one factor $\gamma$ in the integrand of his integral (22), so that actually he has used the following approximation

$$
\frac{K_{0}(t)}{I_{0}(t)} \approx(2 \pi)^{\frac{1}{2}} e^{-2 t}
$$

instead of (24).

In the latter case we find for general values of $z$ and $\rho$

$V(z, \rho) \approx \frac{1}{\left(z^{2}+\rho^{2}\right)^{\frac{1}{2}}}-\frac{1}{a}\left(\frac{8}{\pi}\right)^{\frac{1}{2}} \operatorname{Re}\left\{(2+i \zeta)^{2}-\rho^{2} / a^{2}\right\}^{-\frac{1}{3}}$

leading exactly to Weber's formula for $E_{\rho}$.

In either of the cases Eqs. (24) and (25), the corresponding surface charges, can be calcu- 
lated. We obtained, respectively,

$$
\begin{aligned}
-a^{2} \eta(z) \approx & \frac{1}{4 \pi}\left\{\frac{1}{\left(1+\zeta^{2}\right)^{\frac{1}{2}}}\right. \\
& \left.+\left(\frac{8}{\pi}\right)^{\frac{3}{3}} \operatorname{Re} \frac{3(2+i \zeta)}{(1+i \zeta)^{5 / 2}(3+i \zeta)^{5 / 2}}\right\}, \\
-a^{2} \eta(z) \approx & \frac{1}{4 \pi}\left\{\frac{1}{\left(1+\zeta^{2}\right)^{\frac{3}{2}}}\right. \\
& \left.+\left(\frac{8}{\pi}\right)^{\frac{1}{2}} \operatorname{Re} \frac{1}{(1+i \zeta)^{\frac{1}{3}(3+i \zeta)^{\frac{3}{3}}}}\right\} .
\end{aligned}
$$

Table I shows the three different approximations $\left(23^{\prime}\right),\left(24^{\prime}\right),\left(25^{\prime}\right)$, in presence of the correct values, as computed from (12), (13). The approximation $\left(24^{\prime}\right)$ is obviously very poor, leading to values which are too large at $z \approx 0$ and too small at $z \approx 2 a$. Either of the approximations $\left(23^{\prime}\right)$ and $\left(25^{\prime}\right)$ is excellent. That of Weber $\left(23^{\prime}\right)$ is a better approximation at small values of $z$ than is $\left(25^{\prime}\right)$. On the other hand, for values of $z$ exceeding $a$, the latter is much better than the former. Moreover, Weber's formula yields negative values for $-a^{2} \eta(z)$, when $\zeta>3$.6. This is physically impossible, however. The surface charge density must always have the same sign. The right-hand side of $\left(25^{\prime}\right)$, on the contrary, is positive for large values of $\zeta$. Therefore, as a whole, the approximation (25) may be considered as the most useful one, at least as far as we confine ourselves to the surface charges.

The radial field at the surface of the cylinder, just opposite the point charge, is 1.3262 times as large as it would be under influence of the free-point charge, when the cylinder was removed. The corresponding factors in the three approximations (23), (25), and (24), are 1.3071, 1.3183 , and 1.6142 , respectively.

\section{FORMULAS WHICH ADMIT OF NUMERICAL CALCULATIONS INSIDE THE CYLINDER}

For exact numerical calculations the integral representation (18) is almost without any value. In Section 2 we derived expressions for the charge density which served its useful purpose. The field can be dealt with in a similar manner. The integral (15), however, is no suitable starting point, because its integrand shows a logarithmic singularity in the lower limit. The difficulties owing to the many-valuedness of the $K$-function may be conveniently avoided in the following way. By partially integrating (15) and using (22), one readily obtains

$$
V_{0}(z, 0)=-\frac{2}{\pi z} \int_{0}^{\infty} \frac{\sin (t z / a)}{t I_{0}^{2}(t)} d t .
$$

We now proceed in the same manner as we did before with (5). In this case the path of integration in the complex plane has to avoid the origin by a small indentation in the upperhalf plane. We then obtain for $z>0$

$$
\frac{1}{\pi} \int_{0}^{\infty} \frac{\sin (t z / a)}{t I_{0}^{2}(t)} d t=\frac{1}{2}+\sum_{n=1}^{\infty} \underset{\left(t=i k_{n}\right)}{\operatorname{residue}} \frac{e^{i t z / a}}{t I_{0}{ }^{2}(t)} .
$$

We calculate the residue in $t=i k_{n}$ by putting $t=x+i k_{n}$, developing the function into ascending powers of $x$, and taking the coefficient of $1 / x$ in that expansion. The function is of the type $f(x) / g^{2}(x)$, where $g(x)$ has a simple zero in $x=0$, and $f(0) \neq 0$. The coefficient of $1 / x$ turns out to be

$$
\frac{f}{g^{\prime 2}}\left\{\frac{f^{\prime}}{f}-\frac{g^{\prime \prime}}{g^{\prime}}\right\}_{x=0}=-\frac{z}{a k_{n}} \frac{e^{-k_{n} z / a}}{J_{1}^{2}\left(k_{n}\right)}
$$

Consequently, for $z>0$

$$
V_{0}(z, 0)=-\frac{1}{z}+\frac{2}{a} \sum_{n=1}^{\infty} \frac{e^{-k_{n} z / a}}{k_{n} J_{1}^{2}\left(k_{n}\right)} .
$$

To obtain the total potential on the axis of the cylinder we must add the potential of the unit charge in the origin. For positive values of $z$ the latter is $1 / z$. Hence

$$
V(z, 0)=-\frac{2}{a} \sum_{n=1}^{\infty} \frac{e^{-k_{n} z / a}}{k_{n} J_{1}{ }^{2}\left(k_{n}\right)} .
$$

Applying (16), we finally obtain for $z>0$

$$
V(z, \rho)=-\frac{2}{a} \sum_{n=1}^{\infty} \frac{J_{0}\left(k_{n} \rho / a\right)}{k_{n} J_{1}{ }^{2}\left(k_{n}\right)} e^{-k_{n} z / a}
$$

The series (27) converges also for $x=0$ with the only exception of the origin, where it diverges to infinity. In actual numerical work (27) appears to be useful for $z / a \geqslant 0.2$; in the remaining range $0 \leqslant z / a \leqslant 0.2$ other methods should be applied. 
One naturally asks whether or not it is possible to establish developments for the potential, analogous to that of formula (10) for the surface charge density. Actually, the required transformation is readily performed for points in the axis of the cylinder. We only write down the final result:

$$
\begin{aligned}
& V_{0}(z, 0)=-\frac{2}{\pi z} \sum_{\nu=0}^{\infty} \frac{\sin (\beta \nu+\beta / 2) z / a}{\left(\nu+\frac{1}{2}\right) I_{0}^{2}(\beta \nu+\beta / 2)} \\
& +\frac{1}{a} \sum_{n=1}^{\infty} \frac{\left\{1+e^{-2 \pi k_{n} / \beta}\right\} \cosh \left(k_{n} z / a\right)}{-(2 \pi a / \beta z) \sinh \left(k_{n} z / a\right)} . \\
& k_{n} J_{1}^{2}\left(k_{n}\right) \cosh ^{2}\left(\pi k_{n} / \beta\right)
\end{aligned}
$$

The first series converges for all real $z$, the second for $|z| \leqslant 2 \pi a / \beta ; \beta$ is an arbitrary positive number. If especially $\beta=2 \pi a / z$, the first series vanishes identically, whereas the second one reduces to (27) for $\rho=0$.

For actual numerical evaluation we have chosen $\beta=2$. Then the series (28) becomes

$$
\begin{aligned}
&-a V_{0}(z, 0)=\frac{a}{z}-\{0.79432 \sin (z / a) \\
&+0.01782 \sin (3 z / a)+0.00034 \sin (5 z / a) \\
&\left.+0.00000_{6} \sin (7 z / a)+0.01014 \sinh (2.4 z / a)\right\} \\
& \quad-0.00323 \cosh (2.4 z / a) .
\end{aligned}
$$

Although convergent for $z / a<\pi$, (29) may be useful for $z / a \leqslant 1$.

Unfortunately, there do not seem to exist simple developments for general non-zero values of $\rho$, because (16) yields for the continuation of $(\sin z / a) /(z / a)$ outside the axis

$$
\frac{a}{\rho} \int_{0}^{\rho / a} \cos (t z / \rho) I_{0}(t) d t,
$$

which integral cannot be solved explicitly.

For small values of $z$ we can expand the initial integral (26) into powers of $z / a$. We then obtain

where

$$
V_{0}(z, 0)=-\frac{2}{a} \sum_{\nu=0}^{\infty} \frac{(-1)^{\nu} \lambda_{2 \nu}}{(2 \nu) !}\left(\begin{array}{l}
z \\
a
\end{array}\right)^{2 \nu},
$$

\begin{tabular}{|c|c|c|c|c|c|}
\hline $2 / a \backslash$ & o & 0.2 & 0.4 & 0.6 & 0.8 \\
\hline 0 & $\infty$ & 4.125 & 1.612 & 0.756 & 0.303 \\
\hline 0.1 & 9.131 & 3.600 & 1.539 & 0.736 & 0.297 \\
\hline 0.2 & 4.137 & 2.670 & 1.357 & 0.681 & 0.280 \\
\hline 0.3 & 2.480 & 1.919 & 1.131 & 0.603 & 0.253 \\
\hline 0.4 & 1.661 & 1.395 & 0.916 & 0.518 & 0.224 \\
\hline 0.5 & 1.178 & 1.033 & 0.729 & 0.432 & 0.192 \\
\hline 0.6 & 0.864 & 0.776 & 0.575 & 0.355 & 0.161 \\
\hline 0.7 & 0.648 & 0.591 & 0.410 & 0.287 & 0.133 \\
\hline 0.8 & 0.493 & 0.454 & 0.355 & 0.231 & 0.109 \\
\hline 0.9 & 0.379 & 0.353 & 0.281 & 0.189 & 0.091 \\
\hline 1.0 & 0.292 & 0.272 & 0.219 & 0.147 & 0.071 \\
\hline 1.2 & 0.176 & 0.164 & 0.135 & 0.092 & 0.045 \\
\hline 1.4 & 0.107 & 0.101 & 0.083 & 0.057 & 0.028 \\
\hline 1.6 & 0.066 & 0.062 & 0.051 & 0.036 & 0.017 \\
\hline 1.8 & 0.041 & 0.039 & 0.032 & 0.022 & 0.011 \\
\hline 2.0 & 0.025 & 0.024 & 0.019 & 0.014 & 0.007 \\
\hline
\end{tabular}

$$
\begin{aligned}
\lambda_{2 \nu}= & \frac{1}{(2 \nu+1) \pi} \int_{0}^{\infty} \frac{t^{2 \nu}}{I_{0}{ }^{2}(t)} d t, \\
& \lambda_{0}=0.4353382, \\
& \lambda_{2}=0.2059112, \\
& \lambda_{4}=0.6588568 .
\end{aligned}
$$

TABLE II. Values of the potential (measured in units $1 / a$ ) inside a grounded cylinder under induction of an axial positive unit-point charge; $a=$ radius of the cylinder, $z$ and $p$ the usual cylindrical coordinates. Point charge in the origin.

Except $\lambda_{0}$, these numerical values were given by Watson. ${ }^{5}$

We can now apply (16) with greater success than before. In performing the transformation (16) upon (30) we obtain

$$
V_{0}(z, \rho)=-\frac{2}{a} \sum_{\nu=0}^{\infty} \frac{(-1)^{\nu} \lambda_{2 v}}{(2 \nu) !} F_{\nu}\left(\frac{z}{a}, \frac{\rho}{a}\right),
$$

where

$$
\begin{aligned}
F_{\nu}(u, v)=\frac{1}{2 \pi} \int_{0}^{2 \pi} & (u+i v \cos \varphi)^{2 v} d \varphi \\
= & \left(u^{2}+v^{2}\right)^{\nu} P_{2 \nu}\left\{\frac{u}{\left(u^{2}+v^{2}\right)^{\frac{1}{2}}}\right\},
\end{aligned}
$$

and $P_{2 \nu}$ is the usual symbol. for the Legendre polynomial. For instance

$$
\begin{aligned}
& F_{0}=1, \\
& F_{1}=u^{2}-\frac{1}{2} v^{2}, \\
& F_{2}=u^{4}-3 u^{2} v^{2}+\frac{3}{8} v^{4} .
\end{aligned}
$$

It may be remarked that (32) converges for $z^{2}+p^{2}<4 a^{2}$.

The development (32) becomes much more familiar when spherical coordinates $r, \vartheta$, are introduced, connected to the cylindrical ones by means of

$$
r^{2}=z^{2}+\rho^{2} ; \quad \tan \vartheta=\rho / z .
$$


Then (32) is transformed into

$$
V_{0}(z, \rho)=-\frac{2}{a} \sum_{\nu=0}^{\infty} \frac{(-1)^{\nu} \lambda_{2 \nu}}{(2 \nu) !}\left(\frac{r}{a}\right)^{2 \nu} P_{2 \nu}(\cos \vartheta) .
$$

This series is convergent for $r / a<2$; it is nothing else but the development of $V_{0}(P)$ into spherical potential functions.

Especially in the cut plane $z=0$ we found

$$
\begin{aligned}
-a V_{0}(0, \rho)=0.8707 & +0.1030(\rho / a)^{2} \\
& +0.0206(\rho / a)^{4}+0.0057(\rho / a)^{6} .
\end{aligned}
$$

The first three coefficients are calculated from the values $\lambda_{0}, \lambda_{2}$, and $\lambda_{4}$, as specified above; the last coefficient is taken so that the total is 1.0000 , on account of $V_{0}(0, a)$ being $-1 / a$, thus neglecting terms of higher order than $(\rho / a)^{6}$.

Numerical evaluation of the potential inside the cylinder may be readily accomplished now. The different expressions (27), (28), and (34) have reasonable overlapping regions of useful convergence. Numerical data for the total potential are to be found in Table II.

The electric field itself can also be calculated numerically. From (27) we deduce for $z>0$

$$
\begin{aligned}
& E^{z}=\frac{2}{a^{2}} \sum_{n=1}^{\infty} \frac{J_{0}\left(k_{n} \rho / a\right)}{J_{1}^{2}\left(k_{n}\right)} e^{-k_{n} z / a}, \\
& E_{\rho}=\frac{2}{a^{2}} \sum_{n=1}^{\infty} \frac{J_{1}\left(k_{n} \rho / a\right)}{J_{1}^{2}\left(k_{n}\right)} e^{-k_{n} z / a} .
\end{aligned}
$$

It may be noted that in Watson's paper ${ }^{5}$ two series $S_{1}, S_{2}$ occur which are related to the series (35) by

$$
E_{z}=\frac{2}{a^{2}} S_{1} ; \quad E_{\rho}=-\frac{2}{a} \frac{\partial S_{2}}{\partial z} .
$$

Therefore, Watson's series can be obtained from that shown in (27). Whereas Watson has given explicitly only the first four terms of the development corresponding to (32), we found the general term here expressible in spherical potential functions; cf. (34). With exclusion of the immediate neighborhood of the cut plane $z=0$, Eqs. (35) are very useful for numerical purposes. Around the cut plane we better use (34) and compute $E_{r}, E_{\vartheta}$ instead of the cylindrical components; further details may be omitted, however.

\section{A THIRD METHOD OF SOLUTION}

Either of the two distinct methods developed previously yields complete information concerning the field of the axial point charge in the cylinder. The mathematics there involved are quite instructive, and can serve successfully as a guide in problems of wave propagation inside the same cylinder.

The reader is aware that even in the simplest potential problem, as was treated above, a considerable amount of analysis was used. In this respect wave guide theory is still less tractable. The question whether this is really necessary, from an engineer's point of view, will be answered in due course.

In practical wave guide applications, one is mainly interested in the fields far from the exciting antenna, where many of the higher modes have already been damped out. With this restriction in mind, and thus ignoring the field in the nearby zone, we have investigated whether it is possible to calculate the field in the far zone more directly, without the usual extensive amount of complex function theory. This new method was found by inspection and generalization of the expansion (27), obtained for the potential in the preceding section. Each separate term of that series, for non-vanishing $z$, is a solution of the potential equation, fulfilling the required boundary condition at the surface of the cylinder, and showing the suitable behavior at infinity. So we might have expected in advance the possibility of solving our potential problem by means of such a development, in terms of discrete normal solutions of the potential equation:

$$
V(z, \rho)=\sum_{n=1}^{\infty} c_{n} J_{0}\left(k_{n} \rho / a\right) e^{-k_{n}|z| / a} .
$$

When the proposed method actually works, it must be possible, somehow, to calculate the unknown coefficients $c_{n}$ in this development from the behavior of $V(z, \rho)$ in the immediate neighborhood of the prescribed singularity at the origin, although just in the point charge, (36) is known to diverge. In the following we will show how this problem can be solved. We consider a more general problem, however, so that the new method is equally well applicable to solutions of 
the wave equation. Moreover, we need not restrict ourselves to rotationally symmetrical fields. In'many cases the prescribed singularity is of the type

$$
\frac{1}{R}=\left\{z^{2}+\rho^{2}-2 \rho \rho_{0} \cos \varphi+\rho_{0}^{2}\right\}^{-\frac{1}{3}},
$$

where $R$ denotes the distance between the field point $(z, \rho, \varphi)$ and the source at $\left(0, \rho_{0}, 0\right)$. Singularities of this character are commonly used, both in electrostatics and in wave guide theory. We may, however, admit still more general types of sources.

Suppose $G(z, \rho)$ is a given function with one or more singularities for $z=0,0 \leqslant \rho \leqslant a$. Let $G$ be even in $z$, and let it have continuous derivatives of the second order, outside the singularities. In the previous case of an axial point charge, $G=\left(z^{2}+\rho^{2}\right)^{-1}$.

Furthermore, we suppose that $f_{n}(z)$ is a given system $(n=1,2, \cdots)$ of continuously differentiable functions with the property $f_{n}{ }^{\prime}(0)$ $\neq 0$. For instance, $f_{n}(z)=\exp \left(-k_{n} z / a\right)$.

The problem under consideration can now be stated in the following general form: How to find the coefficients $c_{n}$ in (37), when the following properties of the function $F(z, \rho)$ are known:

(i) $F$ is an even function of $z$.

(ii) $F-G$ has continuous derivatives of the second order, both outside and inside the singularities of $G$.

(iii) For $z \neq 0, F$ can be expanded into a uniformly (in $p$ ) convergent series of the form

$$
F(z, \rho)=\sum_{n=1}^{\infty} c_{n} J_{m}\left(k_{n, m} \rho / a\right) f_{n}(|z|)
$$

where $k_{n, m}$ denotes the $n$th positive zero of the Bessel function $J_{m}(k)$, and $m$ is a given integer.

The answer to this question is readily given if appropriate use is made of the well-known Fourier-Bessel-Dini series theory, for details of which the reader may be referred to Watson's standard work on Bessel functions.

On account of the conditions stated under (ii) we infer that $(\partial / \partial z)(F-G)$ can be developed into a Fourier-Bessel series. This series is uniformly convergent in any closed interval, contained in $0<p<a$, for any value of $z$, whether

${ }^{7}$ G. N. Watson, A Treatise on the Theory of Bessel Functions (Cambridge, 1944), Chapter XVIII. outside or on the cut plane $z=0$. Thus we have

$$
\frac{\partial}{\partial z}(F-G)=\sum_{n=1}^{\infty} b_{n}(z) J_{m}\left(k_{n, m} \rho / a\right) \text {. }
$$

The coefficients $b_{n}(z)$ can be determined in the conventional manner, using the orthogonality of the set of functions $\rho^{\frac{3}{3}} \cdot J_{m}\left(k_{n, m} \rho / a\right)$ upon $0 \leqslant \rho \leqslant a$. One finds?

$b_{n}(z)=\frac{2}{a^{2} J_{m+1}^{2}\left(k_{n, m}\right)} \int_{0}^{a} \rho \frac{\partial}{\partial z}(F-G) J_{m}\left(k_{n, m} \rho / a\right) d \rho$.

As either of the two functions $F, G$ is even in $z$, with continuous derivatives, we have $b_{n}(0)=0$. For points above the cut plane $(z>0)$ the integral may be broken up into two other integrals, namely

$$
\begin{array}{r}
b_{n}(z)=\frac{2}{a^{2} J_{m+1}{ }^{2}\left(k_{n, m}\right)} \frac{\partial}{\partial z} \int_{0}^{a} \rho F(z, \rho) J_{m}\left(k_{n, m} \rho / a\right) d \rho \\
-\frac{2}{a^{2} J_{m+1}{ }^{2}\left(k_{n, m}\right)} \int_{0}^{a} \frac{\partial G}{\partial z} J_{m}\left(k_{n, m} \rho / a\right) d \rho .
\end{array}
$$

From (37), which, for fixed $z \neq 0$ is nothing else but the Fourier-Bessel series of the function $F(z, \rho)$, we obtain

$$
\begin{aligned}
\int_{0}^{a} \rho F(z, \rho) J_{m}\left(k_{n, m} \rho / a\right) & d \rho \\
& =c_{n} f_{n}(|z|) \frac{a^{2}}{2} J_{m+1}{ }^{2}\left(k_{n, m}\right)
\end{aligned}
$$

Therefore, if $z>0$

$b_{n}(z)=c_{n} f_{n}^{\prime}(z)$

$$
-\frac{2}{a^{2} J_{m+1}{ }^{2}\left(k_{n, m}\right)} \int_{0}^{a} \frac{\partial G}{\partial z} J_{m}\left(k_{n, m} \rho / a\right) d \rho .
$$

Now let $z$ tend to zero through positive values; then $b_{n}(z) \rightarrow 0$, and $f_{n}^{\prime}(z) \rightarrow f_{n}^{\prime}(0) \neq 0$. Consequently, the above integral must tend to a limit value also, and the coefficient $c_{n}$ is equal to

$$
\begin{aligned}
c_{n}=\frac{2}{a^{2} f_{n}^{\prime}(0) J_{m+1^{2}}\left(k_{n, m}\right)} \lim _{z \rightarrow 0^{+}} \int_{0}^{a} \frac{\partial G}{\partial z} \\
\times J_{m}\left(k_{n, m} \rho / a\right) d \rho .
\end{aligned}
$$

The actual evaluation of the limit in (38) usually does not lead to difficulties. Let us take 
as an example the potential problem in its simplest form, whereby the point charge is situated on the axis. Then (38) may be applied for $m=0, G=\left(z^{2}+\rho^{2}\right)^{-1}$. In the limit, the main contribution to the integral (38) is attributed to the values of the integrand in the neighborhood of $\rho=0$. Therefore we may substitute unit value for the Bessel function $J_{0}$. Consequently for the limit value

$$
\begin{aligned}
\lim \int_{0}^{a} \frac{\partial}{\partial z}\left(z^{2}+\rho^{2}\right)^{-\frac{1}{2}} d \rho & =\lim \frac{\partial}{\partial z} \int_{0}^{a} \frac{\rho d \rho}{\left(z^{2}+\rho^{2}\right)^{\frac{1}{2}}} \\
& =\lim \frac{\partial}{\partial z}\left\{\left(z^{2}+a^{2}\right)^{\frac{1}{2}}-z\right\}=-1 .
\end{aligned}
$$

Furthermore, $f_{n}{ }^{\prime}(0)=-k_{n, 0} / a=-k_{n} / a$; thus,

$$
c_{n}=\frac{2}{a^{2} k_{n} J_{1}{ }^{2}\left(k_{n}\right)},
$$

in agreement with (27).

Until now we were only interested in boundary-value problems of the first kind, with vanishing $V$ at the surface of the cylinder. In boundaryvalue problems of the second kind, the normal component of $\operatorname{grad} V$ vanishes there; this means $\partial V / \partial \rho=0$ in case of the cylinder. In this second problem the potential can also be developed in terms of Bessel functions. The difference is that the argument $k_{n, m} \rho / a$ is changed into $\kappa_{n, m} \rho / a$, where $\kappa_{n, m}$ means the $n$th positive zero of $J_{m}{ }^{\prime}(\kappa)$. Completely analogous to (37), (38), under similar conditions, we now have the following device:

Given

$$
\Phi(z, \rho)=\sum_{n=1}^{\infty} \gamma_{n} J_{m}\left(\kappa_{n, m} \rho / a\right) f_{n}(|z|),
$$

"behaves like" $G(z, \rho)$ for $z \rightarrow 0$, then the coefficients $\gamma_{n}$ can be determined from

$$
\begin{aligned}
& \gamma_{n}=\frac{2}{a^{2} f_{n}{ }^{\prime}(0)}\left\{1-m^{2} / \kappa_{n, m^{2}}{ }^{2}\right\} J_{m}{ }^{2}\left(\kappa_{n, m}\right) \\
& \times \lim _{z \rightarrow 0^{+}} \int_{0}^{a} \rho \frac{\partial G}{\partial z} J_{m}\left(\kappa_{n, m} \rho / a\right) d \rho .
\end{aligned}
$$

We would like to emphasize that in practical applications we may forget all the precise mathematical conditions and restrictions. Then there only remains the very simple procedure to obtain the coefficients in the postulated expansions (37) and (37') from the Eqs. (38) and $\left(38^{\prime}\right)$, respectively. In this connection the actual evaluation of those limits is of secondary importance.

This third powerful method is incomplete, in so far that it does not very well provide numerical calculations in the nearby zone, because the convergence of the series (37) and (37') is usually too slow there. This, however, might always be overcome by suitable extrapolation of the data outside the cut plane. On the other hand, it has many advantages. For instance, unlike the other two methods, it is equally well applicable to either the potential or the wave equation; the only difference occurs in the system of functions $f_{n}(z)$. Nor does it matter whether or not there is symmetry around the axis of the cylinder.

\section{FIELD OF AN ECCENTRIC POINT CHARGE}

In case of an eccentric point charge, situated at $\left(0, \rho_{0}, 0\right)$, the potential $V(z, \rho, \varphi)$ will be expressible as a Fourier series with respect to $\varphi$, containing only cosine terms. Thus*

$$
\begin{aligned}
V(z, \rho, \varphi)=\sum_{m=0}^{\infty} \epsilon_{m} & \cos m \varphi \sum_{n=1}^{\infty} c_{n, m} \\
& \times J_{m}\left(k_{n, m} \boldsymbol{\rho} / a\right) e^{-k_{n, m}|z| / a}
\end{aligned}
$$

The coefficients $c_{n, m}$ have to be determined from the knowledge that $V$ behaves like $\left(z^{2}+\rho^{2}\right.$ $\left.-2 \rho \rho_{0} \cos \varphi+\rho_{0}^{2}\right)^{-1}$ in the neighborhood of the source at $\left(0, \rho_{0}, 0\right)$. Obviously the method of Section 7 is applicable, if the functions $G, F$, are defined as follows:

$$
\begin{aligned}
G(z, \rho) & \equiv \frac{1}{2 \pi} \int_{-\pi}^{\pi} \frac{\cos m \varphi d \varphi}{\left(z^{2}+\rho^{2}-2 \rho \rho_{0} \cos \varphi+\rho_{0}^{2}\right)^{\frac{2}{2}}} \\
F(z, \rho) & \equiv \frac{1}{2 \pi} \int_{-\pi}^{\pi} V(z, \rho, \varphi) \cos m \varphi d \varphi \\
& =\sum_{n=1}^{\infty} c_{n, m} J_{m}\left(k_{n, m} \rho / a\right) e^{-k_{n, m}|z| / a} .
\end{aligned}
$$

It remains to show how the limit in (38) can be actually evaluated in case of the special $G$-function (40). This can be accomplished by

\footnotetext{
${ }^{*} \epsilon_{0}=1 ; \epsilon_{m}=2$ if $m>0$.
} 
means of a useful symbolic formula, namely

$$
\lim _{z \rightarrow 0^{+}}(\partial G / \partial z)=-\left(1 / \rho_{0}\right) \delta\left(\rho-\rho_{0}\right),
$$

where $\delta$ denotes a delta-function, "vanishing everywhere, except for $\rho=\rho_{0}$, such that its integral over $\rho$ from $-\infty$ to $+\infty$ has unit value." As $G$ is even in $z$, one has, outside the singularity, $\partial G / \partial z \rightarrow 0$ if $z \rightarrow 0$; for $\rho=\rho_{0}$, this derivative tends to infinity. Therefore, the existence of something like (41) seems reasonable. Our final result is completely rigorous, although (41) has only a symbolic meaning. For further details the reader may be referred to the appendix.

When (41) is applied to (38), we easily obtain

$$
c_{n, m}=\frac{2}{a} \frac{J_{m}\left(k_{n, m} \rho_{0} / a\right)}{k_{n, m} J_{m+1}{ }^{2}\left(k_{n, m}\right)},
$$

and thus for the potential of the eccentric charge

$$
\begin{aligned}
& V(z, \rho, \varphi)=\frac{2}{a} \sum_{m=0}^{\infty} \epsilon_{m} \cos m \varphi \\
& \quad \times \sum_{n=1}^{\infty} \frac{J_{m}\left(k_{n, m} \rho_{0} / a\right) J_{m}\left(k_{n, m} \rho / a\right)}{k_{n, m} Y_{m+1}{ }^{2}\left(k_{n, m}\right)} e^{-k_{n, m}|z| / a} .
\end{aligned}
$$

As it should be, this Green function of the first kind for the potential equation inside the cylinder, is symmetrical in $\rho$ and $\rho_{0}$. For $\rho_{0}=0,(42)$ obviously reduces to (27).

The method of the discrete normal solutions has thus proved its value. We could write the proper solution almost at once. Of course, this is not the only way; it is certainly possible to generalize the first or second method, treated in the preceding sections. For instance, one will find for the analog of (5)

$$
\begin{aligned}
\eta(z, \varphi)=-\frac{1}{2 \pi^{2} a^{2}} \sum_{m=0}^{\infty} \epsilon_{m} & \cos m \varphi \\
& \times \int_{0}^{\infty} \frac{I_{m}\left(t \rho_{0} / a\right)}{I_{m}(t)} \cos (t z / a) d t,
\end{aligned}
$$

and by contour integration, analogous to (6),

$$
\begin{aligned}
\eta(z, \varphi)=-\frac{1}{2 \pi a^{2}} \sum_{m=0}^{\infty} \epsilon_{m} & \cos m \varphi \\
& \times \sum_{n=1}^{\infty} \frac{J_{m}\left(k_{n, m} \rho_{0} / a\right)}{J_{m+1}\left(k_{n, m}\right)} e^{-k_{n, m}|z| / a}
\end{aligned}
$$

The same expression for the surface charge density is obtained from (42) if (1) is applied to it.

Similarly, other formulas for the axial point charge can be generalized to an eccentric charge. Further details may be omitted. We better demonstrate the usefulness of the third method by a problem of wave propagation.

\section{THE GREEN FUNCTIONS FOR THE WAVE EQUATION INSIDE THE CYLINDER.$$
\text { RADIATION OF ENERGY BY }
$$$$
\text { ACOUSTIC POINT }
$$$$
\text { SOURCES }
$$

We restrict ourselves to harmonic vibrations of frequency $\omega / 2 \pi$, and time dependence $\exp (-i \omega t)$. The velocity potential of a free-point source is

$$
U_{0}=e^{i k R} / R ; \quad R^{2}=z^{2}+\rho^{2}-2 \rho \rho_{0} \cos \varphi+\rho_{0}{ }^{2} .
$$

Outside the point source, the velocity potential $U$ is a solution of the wave equation

$$
\Delta U+k^{2} U=0 \text {. }
$$

There are two different types of wave functions, according to whether $U$ or $\partial U / \partial \rho$ vanishes at the wall of the cylinder. The Green function of the first kind $U$ is that solution of (44) which vanishes at the surface of the cylinder, which has at infinity the character of plane waves, coming from the direction of the origin of coordinates, and finally such that $U-U_{0}$ remains finite for $R \rightarrow 0$. The Green function of the second kind $U_{2}$ has a vanishing $\partial / \partial \rho$ at $\rho=a$, the other requirements being the same as those for $U_{1}$. Instead of the singularity (43) we may take merely $1 / R$.

Discrete normal solutions of the wave equation (44) are

$$
\begin{aligned}
& \cos m \varphi \cdot J_{m}\left(k_{n, m} \rho / a\right) \\
& \quad \times \exp \left[-(z / a)\left(k_{n, m^{2}}-k^{2} a^{2}\right)^{\frac{1}{5}}\right] \\
& \cos m \varphi \cdot J_{m}\left(\kappa_{n, m} \rho / a\right)
\end{aligned}
$$

$$
\times \exp \left[-(z / a)\left(\kappa_{n, m^{2}}-k^{2} a^{2}\right)^{\frac{1}{2}}\right] .
$$

The functions (45) vanish at $\rho=a$, those of $\left(45^{\prime}\right)$ have a vanishing derivative with respect to $\rho$. On account of symmetry around the plane $\varphi=0$, the potentials do not contain terms with $\sin (m \varphi)$. 
Therefore we may expect for $z \neq 0$

$$
\begin{aligned}
u_{1}=\sum_{m=0}^{\infty} \epsilon_{m} & \cos m \varphi \sum_{n=1}^{\infty} c_{n, m} J_{m}\left(k_{n, m} \rho / a\right) \\
& \times \exp \left[-(|z| / a)\left(k_{n, m^{2}}-k^{2} a^{2}\right)^{\frac{1}{2}}\right], \\
u_{2}=\sum_{m=0}^{\infty} \epsilon_{m} & \cos m \varphi \sum_{n=1}^{\infty} \gamma_{n, m} J_{m}\left(\kappa_{n, m} \rho / a\right) \\
& \times \exp \left[-(|x| / a)\left(\kappa_{n, m^{2}}-k^{2} a^{2}\right)^{\frac{1}{2}}\right] .
\end{aligned}
$$

In these cases the functions $f_{n}(z)$ are, respectively,

$$
\begin{aligned}
& \exp \left[-(z / a)\left(k_{n, m^{2}}-k^{2} a^{2}\right)^{\frac{1}{3}}\right], \\
& \exp \left[-(z / a)\left(\kappa_{n, m^{2}}-k^{2} a^{2}\right)^{\frac{1}{2}}\right] .
\end{aligned}
$$

It is seen that the only difference between (39) and (46) is in the occurrence of different functions $f_{n}(z)$. Consequently we can at once write down the result

$$
c_{n, m}=\frac{2}{a} \frac{J_{m}\left(k_{n, m} \rho_{0} / a\right)}{\left(k_{n, m^{2}}-k^{2} a^{2}\right)^{\frac{1}{3}} J_{m+1}^{2}\left(k_{n, m}\right)} .
$$

Obviously the Green function $U_{1}$ for the wave equation reduces to that of the potential equation (cf. (42)) when $k$ tends to zero. Concerning the square root, we must define for $k a>k_{n, m}$

$$
\left(k_{n, m}{ }^{2}-k^{2} a^{2}\right)^{\frac{1}{2}}=-i\left(k^{2} a^{2}-k_{n, m^{2}}\right)^{\frac{1}{2}}
$$

because of the time factor $\exp (-i \omega t)$.

For the second Green function we obtain by application of $\left(38^{\prime}\right)$

$\gamma_{n, m}=\frac{2}{a} \frac{J_{m}\left(\kappa_{n, m} \rho_{0} / a\right)}{\left(1-m^{2} / \kappa_{n, m}{ }^{2}\right)\left(\kappa_{n, m}{ }^{2}-k^{2} a^{2}\right)^{\frac{1}{2}} J_{m}^{2}\left(\kappa_{n, m}\right)}$,

It is to be noticed that in case of resonance the method fails, because then some coefficient $c$ or $\gamma$ becomes infinitely large. This resonance phenomenon occurs for special values of the applied frequency, namely $k a=k_{n, m}$ or $k a=\kappa_{n, m}$, respectively, for some values of $n, m$. They correspond to the cut-off frequencies of the circular wave guide. In practice actual infinities do not occur, because of certain corrections which have been neglected in our treatment, and which only play an important part near the cut-off frequencies.

Once the amplitudes of the different modes of propagation are known, it is not difficult to compute the amount of energy, radiated by the point source into the cylinder. At large distances from the cut plane $z=0$, the potential is determined by the undamped waves only. So we have

$$
\begin{aligned}
u_{1} \approx \sum_{0<k_{n, m}<k a} \sum_{m} c_{n, m} \cos m \varphi J_{m}\left(k_{n, m} \rho / a\right) \\
\times \exp \left[-i\left(\omega t-(|z| / a)\left(k^{2} a^{2}-k_{n, m^{2}}\right)^{\frac{1}{3}}\right)\right] .
\end{aligned}
$$

Because of the fact that the system of functions

$$
\cos m \varphi J_{m}\left(k_{n, m} \rho / a\right)
$$

is orthogonal over the cross section of the cylinder, one evidently has

$$
\frac{1}{2 \pi} \iint\left|u_{1}\right|^{2} d S=\sum\left|c_{n, m}\right|^{2} \int_{0}^{a} J_{m}^{2}\left(k_{n, m} \rho / a\right) \rho d \rho,
$$

where the surface integral is extended over the cross section of the cylinder, and the summation extends over $n, m$ such that $k_{n, m}<k a$. The integration over $\rho$ can be performed, and after inserting the values of $c_{n, m}$ from (47), one obtains

$$
\begin{aligned}
& \frac{1}{2 \pi} \iint\left|u_{1}\right|^{2} d S \\
& \quad=2 \sum_{0<k_{n, m}<k a} \frac{J_{m}^{2}\left(k_{n, m} \rho_{0} / a\right)}{\left(k^{2} a^{2}-k_{n, m}^{2}\right) J_{m+1}{ }^{2}\left(k_{n, m}\right)}
\end{aligned}
$$

Similarly, for the other problem

$$
\begin{aligned}
& \frac{1}{2 \pi} \iint\left|U_{2}\right|^{2} d S=2 \sum_{0<\kappa_{n}, m<k a} \\
& \times \frac{J_{m}^{2}\left(\kappa_{n, m} \rho_{0} / a\right)}{\left(k^{2} a^{2}-\kappa_{n, m}{ }^{2}\right)\left[1-\left(m^{2} / \kappa_{n, m}\right)\right] J_{m}^{2}\left(\kappa_{n, m}\right)} .
\end{aligned}
$$

The physical meaning of the right-hand side of $\left(48^{\prime}\right)$ is that it provides us with the numeric by which the output energy of a freely radiating source should be multiplied in order to obtain the energy output, when the same source is placed inside the cylinder, at a distance $\rho_{0}$ from the axis.

By the method of images we can also treat the case of a point source inside a cylinder with one end closed. Let $d$ denote the distance from the source to the closed end. Then, by introducing an unclosed cylinder with two sources, a distance $2 d$ apart, and vibrating out or in 
phase, we obtain for the potentials

$$
\begin{aligned}
& u_{1}=U_{1}(z+d, \rho, \varphi)-U_{1}(z-d, \rho, \varphi), \\
& u_{2}=U_{2}(z+d, \rho, \varphi)+U_{2}(z-d, \rho, \varphi) .
\end{aligned}
$$

The far field is a result of two interfering systems of plane waves. It is not difficult to obtain the correspondingly changed energy factors. Before in (48) and $\left(48^{\prime}\right)$ the summation is performed, each separate term must be multiplied by suitable interference factors; these factors are

$$
\begin{aligned}
& 2 \sin ^{2}\left[\begin{array}{l}
d \\
-\left(k^{2} a^{2}-k_{n, m^{2}}\right)^{\frac{1}{2}}
\end{array}\right], \\
& 2 \cos ^{2}\left[\frac{d}{\left.-\left(k^{2} a^{2}-\kappa_{n, m^{2}}\right)^{\frac{1}{2}}\right]}\right.
\end{aligned}
$$

respectively. The resulting expressions, which we need not write down in detail, will then provide us with the numerical factors, by which the output of the free source should be multiplied to obtain its output, when placed at distances $d$ and $\rho_{0}$ from the closed end and the axis, respectively, inside a one-sided cylinder.

Questions of wave propagation in electromagnetic theory can be dealt with in quite the same way, further details of which will not be given at present.

\section{APPENDIX}

The validity of the symbolic formula (41) remains to be proved. That means, in rigorous mathematical terms, we have to prove

$$
\lim _{z \rightarrow 0^{+}} \int_{0}^{a} \rho f(\rho) \frac{\partial G_{m}}{\partial z} d \rho=-f\left(\rho_{0}\right),
$$

where $G_{m}$ is defined by

$$
G_{m}=\frac{1}{2 \pi} \int_{-\pi}^{\pi} \frac{\cos m \varphi d \varphi}{\left(z^{2}+\rho^{2}-2 \rho \rho_{0} \cos \varphi+\rho_{0}^{2}\right)^{\frac{3}{3}}} .
$$

Equation (A) holds true for $0<\rho_{0}<a$, if $f(\rho)$ is continuous upon the closed interval $(0, a)$, but otherwise arbitrary.

For a complete proof of (A) it is sufficient to know that (A) is valid in the following two special cases:

(1) $f(\rho)=\rho^{m}$, where $m$ is the same as in $G_{m}$;

(2) $f(\rho)$ vanishes at $\rho=\rho_{0}$.

The most general case is obviously a linear combination of these special cases, because one can always write

$$
f(\rho)=\frac{f\left(\rho_{0}\right)}{\rho_{0}{ }^{m}} \cdot \rho^{m}+g(\rho),
$$

where $g(\rho)$ vanishes at $\rho=\rho_{0}$.

\section{Case 1}

For $z>0$, from well-known formulas in Bessel-function theory, we have

$$
\begin{aligned}
\left(z^{2}+\rho^{2}-2 \rho \rho_{0} \cos \varphi+\rho_{0}^{2}\right)^{-l} & \\
& =\int_{0}^{\infty} e^{-z t} J_{0}\left[t\left(\rho^{2}-2 \rho \rho_{0} \cos \varphi+\rho_{0}^{2}\right)^{1}\right] d t \\
& =\sum_{0}^{\infty} \epsilon_{\nu} \cos \nu \varphi \int_{0}^{\infty} e^{-z t} J_{\nu}\left(\rho_{0} t\right) J_{\nu}(\rho t) d t .
\end{aligned}
$$

Upon multiplication by $\cos (m \varphi)$, and integration over a full period of $\varphi$, we obtain

Consequently

$$
G_{m}=\int_{0}^{\infty} e^{-z t} J_{m}\left(\rho_{0} t\right) J_{m}(p t) d t .
$$

$$
\begin{aligned}
\int_{0}^{a} \rho \cdot \rho^{m}\left(\partial G_{m} / \partial z\right) d \rho & \\
& =\int_{0}^{a} \rho^{m+1} d \rho \int_{0}^{\infty}-t e^{-z t} J_{m}\left(\rho_{0} t\right) J_{m}(\rho t) d t \\
& =-\int_{0}^{\infty} t^{-m-1} e^{-z t} J_{m}\left(\rho_{0} t\right) d t \int_{0}^{a t} \kappa^{m+1} J_{m}(x) d x \\
& =-a^{m+1} \int_{0}^{\infty} e^{-z t} J_{m}\left(\rho_{0} t\right) J_{m+1}(a t) d t .
\end{aligned}
$$

Let $z$ tend to zero; then the integral above tends to

$$
\int_{0}^{\infty} J_{m}\left(\rho_{0} t\right) J_{m+1}(a t) d t=\left(\rho_{0}^{m} / a^{m+1}\right) \quad\left(0<\rho_{0}<a\right) .
$$

Hence

$$
\lim _{z \rightarrow 0^{+}} \int_{0}^{a} \rho \cdot \rho^{m}\left(\partial G_{m} / \partial z\right) d \rho=-\rho_{0^{m}}
$$

which is (A) for the special function $f(\rho)=\rho^{m}$.

\section{Case 2}

It is easy to demonstrate that the contribution of the intervals outside the immediate vicinity of $\dot{\rho}_{0}$ is zero; that means

$$
\lim _{z \rightarrow 0^{+}}\left\{\int_{0}^{\rho_{0}-\epsilon} \rho f(\rho) \frac{\partial G_{m}}{\partial z} d \rho+\int_{\rho_{0}+\epsilon}^{a} \rho f(\rho) \frac{\partial G_{m}}{\partial z} d \rho\right\}=0,
$$

where $\epsilon$ is any positive constant, such that $0 \leqslant \rho_{0}-\epsilon<\rho_{0}$ $+\epsilon \leqslant a$. For the proof of $(\mathrm{E})$ we introduce $M$, the upper bound of $|f(\rho)|$ upon $(0, a)$. Then

$$
\left|\int_{0}^{\rho_{0}-\epsilon} \rho f(\rho) \frac{\partial G_{m}}{\partial z} d \rho\right| \leqslant M \int_{0}^{\rho_{0}-\epsilon} \rho\left|\frac{\partial G_{m}}{\partial z}\right| d \rho .
$$

Furthermore,

$$
\frac{\partial G_{m}}{\partial z}=-\frac{z}{2 \pi} \int_{-\pi}^{\pi} \frac{\cos m_{\varphi} d \varphi}{\left(z^{2}+\rho^{2}-2 \rho \rho_{0} \cos \varphi+\rho_{0}^{2}\right)} ;
$$

thus

$$
\left|\frac{\partial G_{m}}{\partial z}\right| \leqslant \frac{z}{2 \pi} \int_{-\pi}^{a \pi} \frac{d \varphi}{\left(z^{2}+\rho^{2}-2 \rho \rho_{0} \cos \varphi+\rho_{0}^{2}\right)^{1}} \leqslant \frac{z}{\left[z^{2}+\left(\rho-\rho_{0}\right)^{2}\right]^{3}} .
$$

Therefore,

$$
\left|\frac{\partial \grave{G}_{m}}{\partial z}\right| \leqslant \frac{z}{\left(z^{2}+\epsilon^{2}\right)^{1}} \text { if } \quad\left|\rho-\rho_{0}\right| \geqslant \epsilon .
$$

Consequently

$$
\left|\int_{0}^{\rho_{0}-\epsilon} \rho f(\rho) \frac{\partial G_{m}}{\partial z} d \rho\right| \leqslant \frac{M z}{\left(z^{2}+\epsilon^{2}\right)^{3}} \int_{0}^{\rho_{0}} \rho d \rho .
$$


A similar bound is found for the contribution of the interval $\left(\rho_{0}+\epsilon, a\right)$. Upon combining them we obtain

$$
\left|\int_{0}^{\rho_{0}-\epsilon} \rho f(\rho) \frac{\partial G_{m}}{\partial z} d \rho+\int_{p_{0}+\epsilon}^{a} \rho f(\rho) \frac{\partial G_{m}}{\partial z} d \rho\right| \leqslant \frac{M z q^{2}}{2\left(z^{2}+\epsilon^{2}\right)^{!}} .
$$

Let now $z$ tend to zero, $\epsilon$ being kept constant. Then $(E)$ follows at once. (It should be noted that (E) holds for general functions $f(\rho)$, not necessarily vanishing at $\rho_{0}$.)

For the special function $f(\rho)$, with $f\left(\rho_{0}\right)=0$, the contribution of the remaining interval $\rho_{0}-\epsilon \leqslant \rho \leqslant \rho_{0}+\epsilon$ is very small, because $f(p)$ is very small there. Let us introduce the upper bound of $\{f(\rho)\}$ in this interval, thus

$$
\left.M(\epsilon)=\operatorname{Max}_{\left|\boldsymbol{\rho}-\boldsymbol{\rho}_{0}\right| \leqslant \epsilon} \mid f(\boldsymbol{\rho})\right\},
$$

then obviously $M(\epsilon) \rightarrow 0$ if $\leftrightarrow \rightarrow 0$. Furthermore,

$$
\left|\frac{\partial G_{m}}{\partial z}\right| \leqslant \frac{z}{2 \pi} \int_{-\pi}^{+\pi} \frac{d \varphi}{\left(z^{2}+\rho^{2}-2 \rho \rho_{0} \cos \varphi+\rho_{0}^{2}\right)^{1}}=-\frac{\partial G_{0}}{\partial z} .
$$

Therefore,

$$
\begin{aligned}
\left|\int_{\rho_{0-\epsilon}}^{\rho_{0}+\epsilon} \rho f(\rho) \frac{\partial G_{m}}{\partial z} d \rho\right| \leqslant-M(\epsilon) \int_{\rho_{0}-\epsilon}^{\rho_{0}+\epsilon} \frac{\partial G_{0}}{\partial z} d \rho & \\
& \leqslant-M(\epsilon) \int_{0}^{a} \rho \frac{\partial G_{0}}{\partial z} d \rho .
\end{aligned}
$$

Let now $z$ tend to zero; then the right-hand integral tends to -1 , as follows from (D) when the latter formula is applied with $m=0$.
Thus we have

$$
\left|\lim _{z \rightarrow 0^{+}} \int_{\rho 0^{-\epsilon}}^{\rho_{0}+\epsilon} \rho f(\rho) \frac{\partial G_{m}}{\partial z} d \rho\right| \leqslant M(\epsilon) ;
$$

that means, on account of $(\mathrm{E})$

$$
-M(\epsilon) \leqslant \lim _{z \rightarrow 0^{+}} \int_{0}^{a} \rho f(\rho) \frac{\partial G_{m}}{\partial z} d \rho \leqslant M(\omega) .
$$

This is true for any $\epsilon>0$; as $M(\epsilon) \rightarrow 0$ for $\leftrightarrow 0$, consequently

$$
\lim _{z \rightarrow 0^{+}} \int_{0}^{a} \rho f(\rho) \frac{\partial G_{m}}{\partial z} d \rho=0=-f\left(\rho_{0}\right)
$$

and this is the required expression in case 2 . So we have rigorously proved the validity of the symbolic formula (41). It may be noticed here that according to Watson ${ }^{8}$

${ }^{8} \mathrm{G}$. N. Watson, reference 7, page 389 , Eq. (2). we can deduce from $(C)$ the following interesting formula

$$
G_{m}=\frac{1}{\pi\left(\rho \rho_{0}\right)^{3}} Q_{m-3}\left(\frac{z^{2}+\rho^{2}+\rho_{0}^{2}}{2 \rho \rho_{0}}\right) \text {, }
$$

where $Q$ denotes the Legendre function of the second kind. The $Q$-function has a singularity at the point where the argument is equal to 1 ; this corresponds with $z=0, \rho=p_{0}$.

Eindhoven, March, 1946. 